\title{
The Analysis with Finite Elements of the Elasto-plastic Behaviour of the Spinal Immobilizers - in the Case of Comminutive Fractures
}

\author{
BOGDAN FLORIN TOMA ${ }^{1,2}$, CONSTANTIN NANU ${ }^{3}$, CEZAR POPESCU $^{4}$, \\ RAZVAN VLADIMIR SOCOLOV ${ }^{2,5}$, VASILE- EDUARD ROSU ${ }^{6,7}$, STEFAN LUCIAN TOMA $^{8 *}$, \\ LOREDANA MARIA HIMINIUC ${ }^{1,2 *}$, SOLANGE TAMARA ROSU ${ }^{6,9}$ \\ ${ }^{1}$ Cuza Voda Obstetrics and Gynecology Clinical Hospital, 34 Cuza Voda Str., 700038, Iasi, Romania \\ ${ }^{2}$ Gr. T. Popa University of Medicine and Pharmacy Iasi, Department of Obstetrics and Gynecology, 16 University Str, \\ 700115, Iasi, Romania \\ ${ }^{3}$ Clinical Emergency Hospital, 1 Traian Blvd, 610136, Piatra Neamt, Romania \\ ${ }^{4}$ Prof. Dr. Nicolae Oblu Emergency Clinic Hospital, 2 Ateneului Str., 700309, Iasi, Romania \\ ${ }^{5}$ Elena Doamna Obstetrics and Gynecology Clinical Hospital, 49 Elena Doamna Str., 700398, Iasi, Romania \\ ${ }^{6}$ Sf. Maria Emergency Pediatric Hospital, 62 Vasile Lupu Str., 700309, Iasi, Romania, \\ ${ }^{7}$ Gr. T Popa University of Medicine and Pharmacy, Departament of Mother and Child Medicine, 16 University Str., 700115, \\ Iasi, Romania \\ ${ }^{8}$ Gheorghe Asachi Technical University of Iasi-Romania, Department of Materials Engineering and Industrial Safety, 43 \\ Prof. dr. doc. Dimitrie Mangeron Blvd., 700050, Iasi, Romania \\ ${ }^{9}$ Gr. T Popa University of Medicine and Pharmacy, Departament of Medical Specialities II, 16 University Str, 700115, Iasi, \\ Romania
}

\begin{abstract}
In this paper it is presented a comparative theoretical study - performed by finite element analysis (FEA), of the tension state that appears at the level of the spine, as well as in the areas adjacent to it, under the following conditions: a) the existence of a comminutive fracture at the level of the vertebra $T 11 ; b)$ of the external immobilization of the body through a Lombax orthesis $(L O)$ or of the internal immobilization of the column by means of a spinal fixator $(S F)$; $c$ ) of performing some basic flexion or extension movements. In this study, both flexural force and extension force varied on three levels. The results of the comparative theoretical study were supplemented with the clinical observations obtained from a number of 52 patients who had suffered comminutive fractures in the T11 vertebra and who were treated - by external immobilization, in the Lombax orthesis or were surgically treated - by applying a trapped spinal fixator on the T10-T12 vertebrae. The study had demonstrated that, by immobilizing the body in the Lombax orthesis, following the application of the flexion or extension movement, the mobility of the body is low, the bone fragments move less distances by about $12 \%$ against to their displacement under the condition of immobilizing the column by a spinal fixator. Although, in the latter case, the displacements of the bone fragments are greater, the elastic behaviour of the spinal fixator determines the returning of the fragments and of the spine to the initial position as well as the distance between the vertebrae. Clinical investigations on patients treated by external immobilization of the body in the Lombax orthesis show that the values of the local kyphosis angle (LKA) are about 30\% lower than the values of the same parameter obtained under the surgical treatment.
\end{abstract}

Keywords: Spine, Lombax orthesis, internal fixator, finite element

\section{Introduction}

Also called "burst fractures", comminutive fractures are injuries to the spine through which a vertebra breaks into small pieces - from a high-energy task (traffic collision, falls from heights, sports activities, etc.) which penetrate the spinal canal and in the surrounding tissues, [1]. Most commonly, comminutive fractures occur in the thoraco-lumbar zone. This region of the spine is a vulnerable area because it is a junction between the thoracic column - rigid and the lumbar - mobile column. In most cases, fractures produced at the vertebral body level are stable and aren't accompanied by neurological deficits. The

\footnotetext{
*email: stltoma@gmail.com,loredanahiminiuc@gmail.com
} 
main treatment methods frequently applied in the case of burst fracture of the spine are based either on external immobilization of the body in orthesis or surgical - by implanting a spinal fixator (SF) on vertebrae adjacent to the fracture. Due to the reduced complexity and low price, the method of treatment based on the outer fixation of the body in the Lombax orthesis (LO) - made of plastic material with metal insert (to be light and elastic) is the most known and used method. The inconvenience generated by the wearing of the orthesis and the long time it takes for the complete restoration of the traumatized vertebra make the surgical treatment to be the preferred one for the burst fractures of the spine. The devices for spinal fixation devices are rigidly attached to the vertebrae adjacent to the fractured vertebrae - by means of two pedicular screws and allow the bone replacement, the restoring of the alignment, the maintaining positions and the preventing the movement in the treatment of fractures, of degenerative diseases and congenital deformities, [2,3]. However, surgery for comminutive fractures involves a high risk of occurrence post-operative complications, long recovery time - lower than in the case of applying the treatment method based on immobilization in orthesis [4], and a certain functional status of the patients, characterized by the absence of affections of diabetes type, ischemic cardiopathy, renal etc.

Finite Element Analysis is a method frequently used by various researchers in biomechanics [5-8] to study the state of tension and the behaviour of the spine in different conditions of stress. The models created of the spine are simple, consisting of 3-4 vertebrae [9] and the theoretical results obtained are compared with experimentally obtained results - under the same conditions [10]. Thus, Manwan El-Rich et colab. [5] demonstrated that the sudden movement by flexion or extension in sagittal plane generates high tensions at the vertebrae level and in the intervertebral discs. F. Heuer et colab. [11], demonstrated that these stresses are also included at the ligaments level afferent to the spine.

In this paper, a comparative theoretical study was carried out of the effect created by the elastoplastic behaviour of the spinal fixator, respectively of the Lombax orthesis, on the local displacements of the vertebrae fragments from a comminutive fractured vertebra, as well as on the adjacent area of it.

The results of this study are useful in establishing a sustainable treatment method. The comparative theoretical study, carried out by the Finite Element Analysis Method (FEA), allowed the determination of local displacements of the fractured vertebrae and of the total von Mises tensions, existed in the adjacent area of the fractured vertebra - in the case of execution the basic movements: flexion and extension in sagittal plane. The theoretical results of the study were supplemented with observations and clinical investigations performed groups of patients who had suffered burst fractures of the spine and who were treated by both methods.

\section{Materials and methods}

\section{Finite element analyses of study}

To analyse comparatively the local displacements of the vertebral fragments and the existing state of tension at the level of the fractured vertebrae in the condition of applying the two types of treatments and of the execution of basic movements: flexion or extension, the Finite Element Analysis method (FEA) was used, respectively the Ansys Multiphysics program [11]. For this purpose, a two-dimensional model of the body (Figure 1), which contain a fracture of burst fracture type was made - in the lumbar area at T11 vertebra level and which was immobilized by external fixation in the LO (Figure1a), respectively by internal fixation, by means of a spinal fixator (SF) disposed at the adjacent vertebrae level: T10 and T12 (Figure 1b). The considered model contains: vertebrae T1-T12 and L1-L5 formed from cortical bone (CB) and Cancellous bone (CCB), intervertebral discs and ligaments: anterior longitudinal ligament (ALL), posterior longitudinal ligament (PLL), ligamentum flavum (LF); interspinous ligament (ISL), supraspinous ligament (SSL). Intervertebral discs - figure 1, were considered to be an assembly composed of: pulpous nucleus (NS) - made from an incompressible fluid field; annular fibrosis (AS) - made of a hyperplastic composite material, fibre reinforced; cartilaginous endplate (EP) - made of a hyperplastic composite material similar to the model made by Rohlmann and colab., $[13,14]$. 

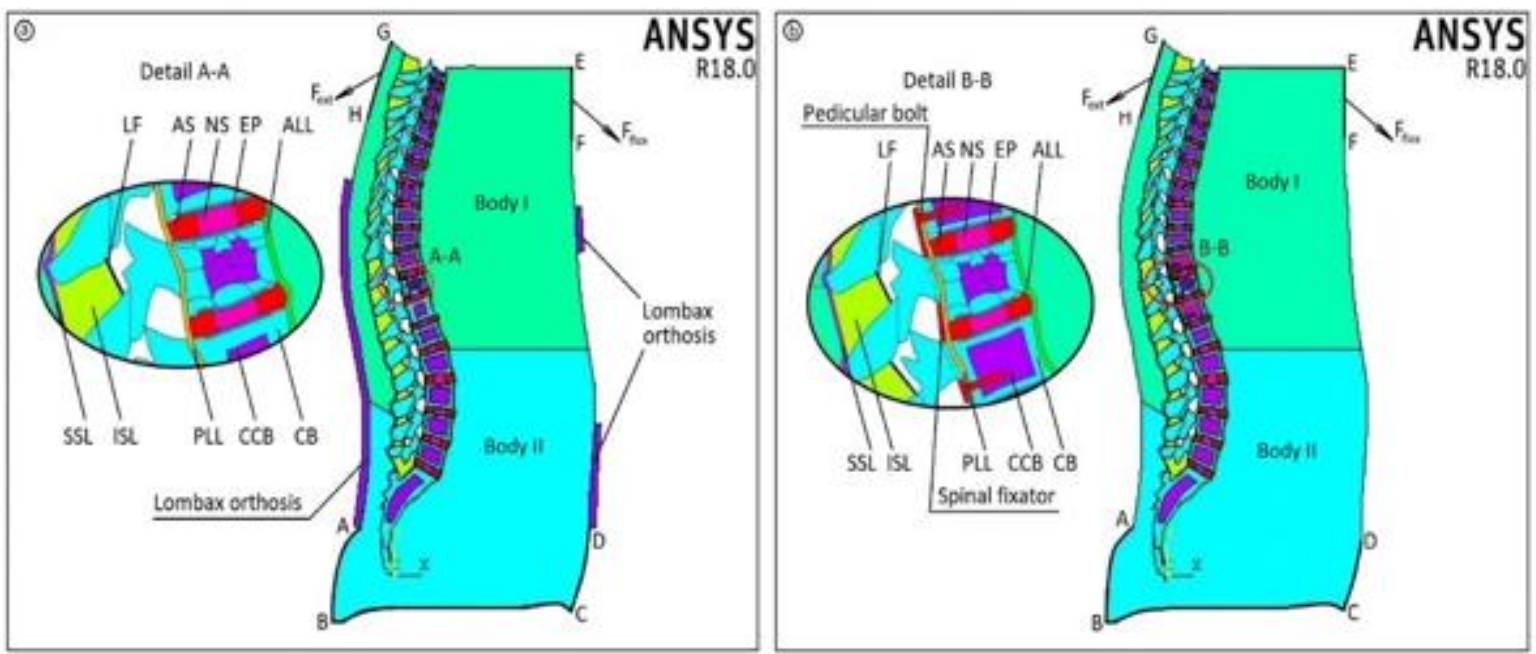

Figure 1. Models used in finite element analysis: (a) fracture model stabilized using Lombax orthesis;

(b) fracture model stabilized by means of a spinal fixator - unilateral fixation: ALL - anterior longitudinal ligament; PLL - posterior longitudinal ligament; LF - ligamentum flavum; ISL interspinous ligament; SSL - supraspinous ligament; CB - cortical bone CCB - Cancellous bone; AN - annulus; NS - nucleus; EP - endplate; detail A-A and BB - T11 vertebra

The spine vertebrae were constructed in longitudinal axial section, made after computerized topographies of the spine performed on a male patient of 21-year-old - similar to the model proposed by Nanu and colab., [6]. The spinal fixator (Figure 1b), was considered to be made of titanium and attached by means of two pedicular screws to the vertebrae body T10 and T12 - in the posteriors part of the spines. It was considered that the materials used have the physical properties isotropic and homogeneous and the dates were taken over from the speciality technical literature, [14-20] (Table 1).

The ligaments were considered to be viscous - elastic and isotropic, for their modelling we used the elastic elements available in the ANSYS program. The section plan, chosen to represent the model, doesn't include the chest box and the abdomen.

Its homogeneous and isotropic behaviour has been simplified by attributing different properties to materials: Body I and Body II (Table 1), [5, 21]. All elements of the model were discretized with solid two-dimensional elements such as: plane 82, plane 183 and plane 192 (Table 2), resulting in a total of 205808 elements and a number of 663975 nodes. The faces of the articulations have been treated as a contact problem nonlinear, two-dimensional, without friction.

Table 1. Material properties used in fea of the lumbar spine [5, 9, 10, 12, 14-20, 23-28]

\begin{tabular}{|c|c|c|c|c|}
\hline Material & $\begin{array}{c}\text { Young's Modulus, } \\
\text { (MPa) }\end{array}$ & $\begin{array}{c}\text { Poisson } \\
\text { ratio }\end{array}$ & $\begin{array}{c}\text { Density } \\
{\left[\mathrm{Kg} / \mathbf{m m}^{3}\right]}\end{array}$ & $\begin{array}{c}\begin{array}{c}\text { Cross section } \\
\left(\mathbf{m m}^{2}\right)\end{array} \\
\end{array}$ \\
\hline \multicolumn{5}{|c|}{ Vertebra } \\
\hline Cortical bone & 12000 & 0.3 & $1.83 \mathrm{E}-06$ & - \\
\hline Cancellous bone & 200 & 0.2 & $0.17 \mathrm{E}-06$ & - \\
\hline Posterior bony elements & 3500 & 0.25 & $1.06 \mathrm{E}-06$ & - \\
\hline \multicolumn{5}{|l|}{ (1) } \\
\hline Annulus & 8.4 & 0.45 & $1.20 \mathrm{E}-06$ & - \\
\hline Nucleus & 1.0 & 0.495 & $1.0 \mathrm{E}-06$ & - \\
\hline Endplate & 24.0 & 0.4 & & - \\
\hline \multicolumn{5}{|c|}{ Ligament } \\
\hline ALL & 19.2 & 0.4 & $1.0 \mathrm{E}-06$ & 63.7 \\
\hline PLL & 84.1 & 0.4 & $1.0 \mathrm{E}-06$ & 20 \\
\hline $\mathrm{LF}$ & 4.2 & 0.4 & $1.0 \mathrm{E}-06$ & 40 \\
\hline ISL & 4.7 & 0.4 & $1.0 \mathrm{E}-06$ & 40 \\
\hline SSL & 10.2 & 0.4 & $1.0 \mathrm{E}-06$ & 30 \\
\hline
\end{tabular}




\begin{tabular}{cccc} 
Body I & 70540 & 0.342 & - \\
Body II & 60210 & 0.38 & - \\
& \multicolumn{1}{c}{ Other elements } & 7,8 \\
$\begin{array}{c}\text { Plastic and stainless steel } \\
\text { (Lombax orthesis) } \\
\begin{array}{l}\text { Titanium alloy } \\
\text { (spinal fixator) }\end{array}\end{array}$ & 210000 & 0.33 & 4.55 \\
\hline ALL - anterior longitudinal ligament; PLL - posterior longitudinal ligament; LF - ligamentum flavum; ISL - interspinous \\
ligament; SSL - supraspinous ligament.
\end{tabular}

Table 2. Element type used in the fea of the spine

\begin{tabular}{ccc}
\hline Intervertebral body & & Element type \\
\hline Vertebrae & Cortical bone, Cancellous bone & Plane 183 \\
Disc & Annulus, Nucleus, Endplate & Plane 82 \\
Ligament & ALL, PLL, SSL & Plane 192 \\
& LF, ISL & Shell 192 \\
Body & Body I, Body II & Plane 183 \\
Orthesis Lombax & Plastic material & Plane 183 \\
Spinal fixator & Spinal instrumentation & Plane 183 \\
\hline
\end{tabular}

To validate the model, we considered an area adjacent to the T11vertebra, respectively the thoracolumbar assembly formed by the vertebrae $\mathrm{T} 12, \mathrm{~L} 1$, the intervertebral disc $\mathrm{T}_{12}-\mathrm{L}_{1}$ and ligaments to which we determined the interdiscal pressure (IDP), in the condition in which the vertebra L1 is fixed and on the vertebra T12 acts a pure rotational moment - in xOy plane that simulates the flexion movement or extension (Figure 2). Similar to the work developed by Goel V.K. et al. [22], the assembly was loaded successively with pure moments of rotation of 1, 2,5, 5, 7.5 and $10 \mathrm{Nm}$ (M+ for flexion, $\mathrm{M}$ - for extension) and in each case the pressure exerted in the intervertebral disc $\mathrm{T}_{12}-\mathrm{L}_{1}$ was calculated, considered as one among the most important parameters for evaluating the disc and estimating intervertebral stress [23] Figure 2. The results of the analytical calculations performed were compared with the results obtained in vivo by Heurer et al. [24] and of Schmidt et al. [25]. In order to be consistent with the in vivo experimental determinations, the simulations were performed under the following limit conditions: the surfaces inside the model that represented the soft tissues were removed - figure 2, between the surfaces in contact was considered zero friction force, vertebra L1 was considered fixed at the level of the lower surface - along the contour line ABCD.

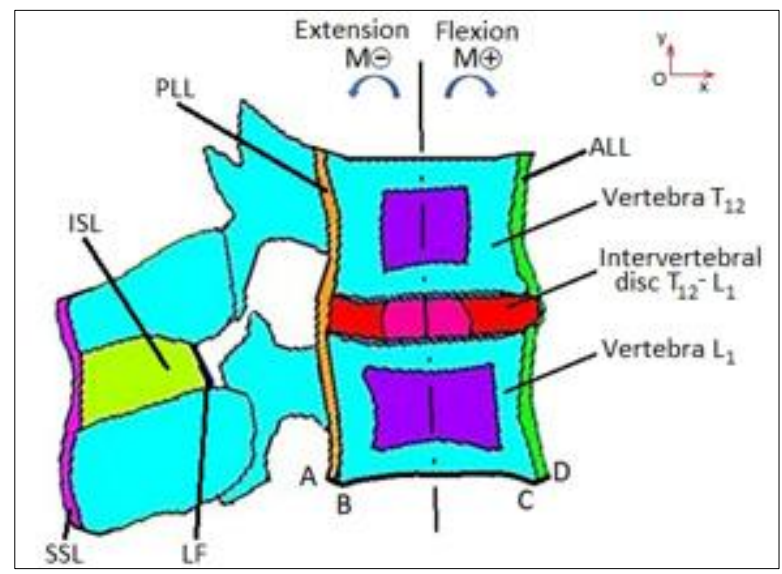

Figure 2.

Flexion/extension

loading of the

thoraco-lumbar

assembly

The calculation hypothesis has aimed the analytical calculation of the maximum interdiscal pressure that manifests at the level of the intervertebral disc $\mathrm{T}_{12}-\mathrm{L}_{1}$ in the condition that on the T12 vertebra is exerted a moment of pure rotation - which varies successively on 5 levels, necessary to simulate the flexion movement or extension. The characteristics of the materials involved in the validation are presented in Table 1. 
In Figure 3 there are comparatively presented the values of the IDP the results by simulation and the values of the interdiscal pressure determined in vivo. As expected, by increasing the applied momentum, the IDP increases both in the case of flexion movement and in the case of extension movement - similar to the in vivo variation of the interdiscal pressure. The IDP values obtained by calculation, at the execution of the flexion movement are up to 50\% higher than the IDP values obtained at the execution of the extension movement (Figure 3a vs. 3b). The large differences between the two values were obtained at the value of $5 \mathrm{Nm}$ of the rotation moment. It can be seen that, the values obtained for IDP both in the condition of performing the flexion and the extension movement, are in the domain of standard deviations of each in vivo determination - aspect that validates the considered model.

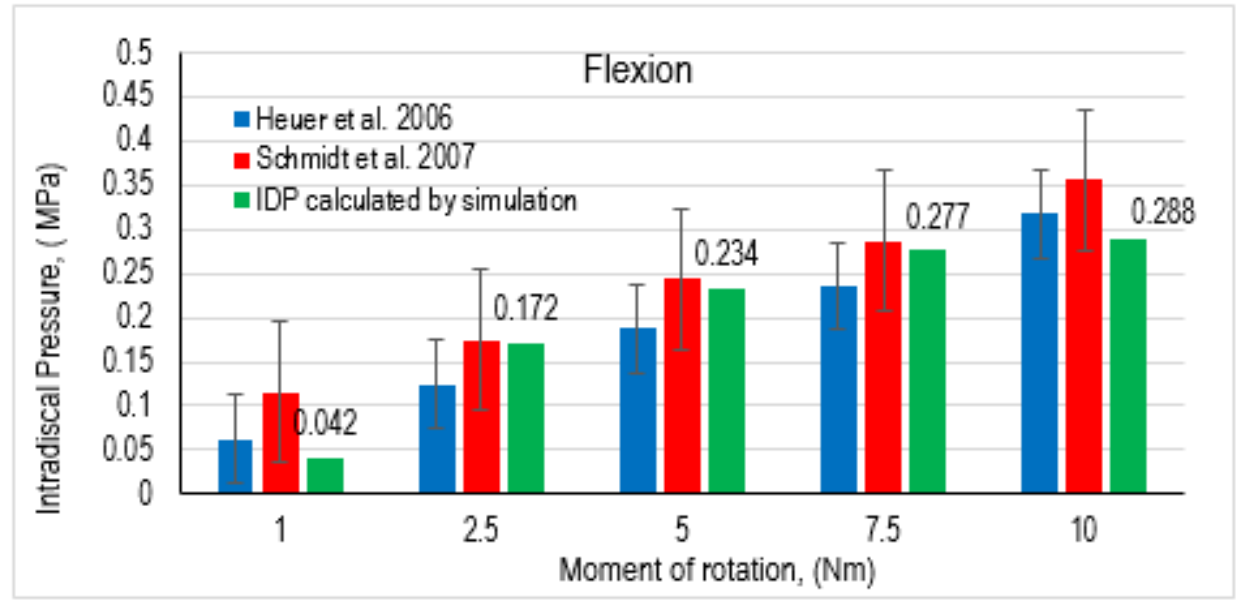

a)

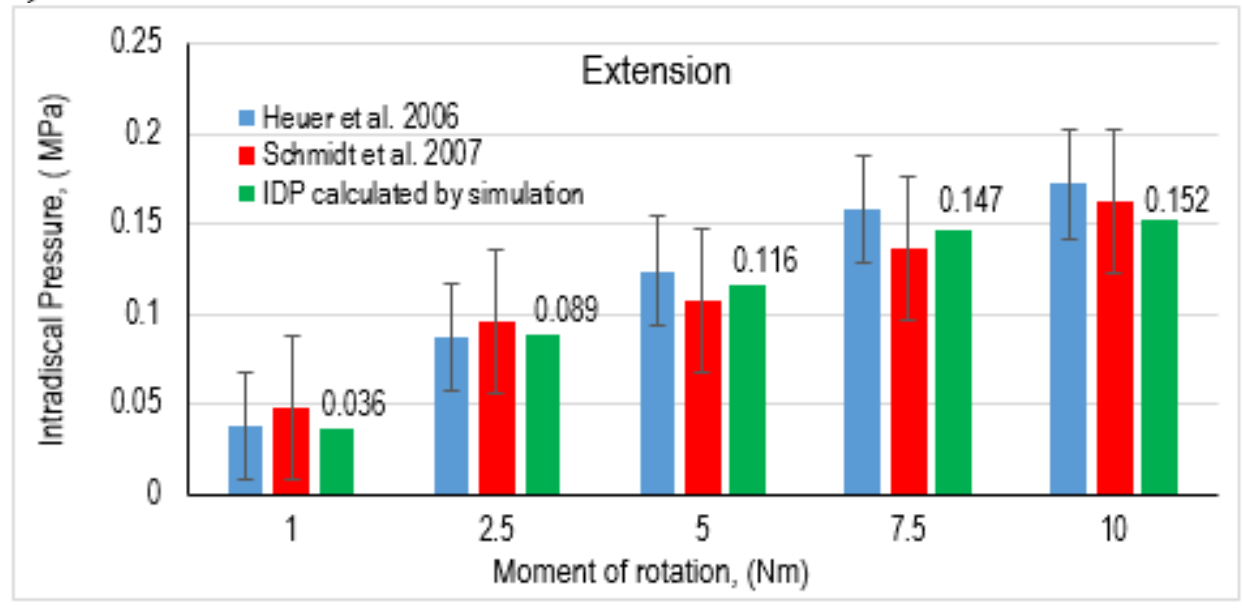

b)

Figure 3. Comparatively, the IDP values obtained by flexion/extension simulation and the IDP values measured in vivo by Heurer et al. [24] and Schmidt et al. [25]: a) flexion; b) extension, (vertical bars represent the standard error)

To be in concordance with the kinematics and the charge of the column in vivo, elementary positions such as: the flexion and the body extension specific to the activities normally performed in everyday life were simulated.

In both cases of spine stabilization, the following boundary conditions were taken into account in the simulation: at the contact between the joints of the faces were considered contact elements surface-tosurface without friction, the pattern was rigidized along the A-B-C-D contour and subjected to a flexion $\left(\mathrm{F}_{\text {flex }}\right)$, respectively to an extension force $\left(\mathrm{F}_{\text {ext }}\right)$ - applied along the lines $\mathrm{EF}$ and $\mathrm{GH}$ - see figure-1, inclined at an angle of $60^{\circ}$ to the direction of the longitudinal axis of the body. These forces were necessary to simulate the flexion movement, respectively the extension of the body. In the theoretical study realized, the values of the flexion and extension force varied on three levels (Table 3). Using the ANSYS 
programme we calculated: the displacement of the bone fragments 1-6 components of the fractured vertebra (Figure )4, the value of von Mises total tension - in the area adjacent to the fractured vertebra T11 and the amplitude of the movement, for each experiment.

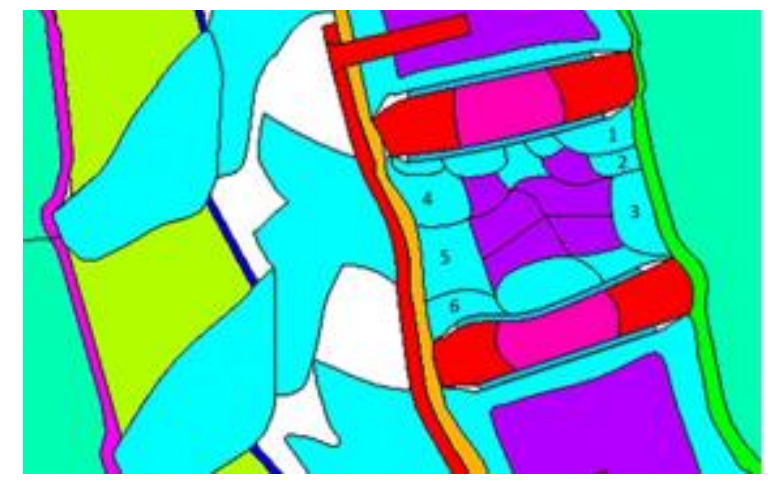

Figure 4. Vertebral fragments of the fractured vertebra $\mathrm{T} 11$

In the simulations performed, the friction force that occurs at the contact between the bone fragments of the vertebra T11 has been neglected and the fragments bone were considered to be rigid.

Table 3. Simulation program

\begin{tabular}{cccc}
\hline Immobilization type & Motion type & $\begin{array}{c}\text { Force, } \\
(\mathbf{N})\end{array}$ & $\begin{array}{c}\text { Amplitude of movement, } \\
{[\mathbf{m m}]}\end{array}$ \\
\hline Outside: Lombax orthesis (LO) - & Flexion & $300 / 500 / 700$ & $122 / 126 / 128$ \\
external fixation & Extension & $300 / 500 / 700$ & $98 / 102 / 106$ \\
Internal: spinal fixator (SF) - internal & Flexion & $300 / 500 / 700$ & $138 / 141 / 146$ \\
fixation & Extension & $300 / 500 / 700$ & $104 / 106 / 111$ \\
\hline
\end{tabular}

\section{Clinical study of fracture}

At the Clinical Emergency Hospital Piatra Neamt, were developed clinical observations on a number of 32 patients, who have sustained fractures at the T11 vertebra - burst fracture type, which had the local segmental kyphosis angle lower than $30^{\circ}$ and they weren't presenting neurological deficits. Patients with pathological fractures, initial neurologic deficit, posterior ligamentous lesions, the angle of kyphosis higher than 30, polytrauma, were excluded from this study. The patients were divided into two groups: group I - consisted of 18 conservatively treated patients - by external immobilization with a thoracolumbar orthesis of Lombax type; group II - consisted of 14 patients treated surgically - by applying an internal spinal fixation on the T10-T12 vertebra. The decision for the surgical treatment of patients belonged to the treating physician - being determined by the comminution severity degree, according to the Load Sharing Classification (11 patients) or at their request ( 7 patients).

Both patient groups were periodically evaluated at 3,6 and 12 months by performing of standard radiographical analyses in orthostatism. On the profile images it was calculated the angle of segmentation kyphosis between the inferior plate of lower vertebra and the superior plate of the proximal vertebra using the Cobb method. The pain was evaluated by the VAS scale (Visual Analogue Scale) and the functional status - indicator of spinal column mobility was evaluated using the ODI scale (Oswestry Disability Index).

\section{Results and discussions}

This study presents the results in two parts: in the first part are presented the results obtained after the analysis with finite elements of the stress state, the deformation and the displacements of bone fragments from the fractured vertebra T11 - in the conditions exposed in Table 3 and in the second part there are presented the observations results and the clinical investigations performed on the groups of patients presented in clinical study. 


\section{Biomechanical analysis of the two FEA models}

Using the models presented in Figure 1, the state of tension, elastic deformations and local displacements of fragments of the fractured vertebra T11 were determined, in the condition of executing movements of flexion, respectively extension.

In Figure 5 are presented the total variation of the von Mises stresses - inside the body, respectively at the fractured vertebra T11 level, in the condition of executing a movement of flexion.

In Figure 6 is presented the variation of von Mises total tensions inside the body, respectively at the level of the fractured vertebra T11, in the condition of execution an extension movement. At low values of the flexion force - in the condition of immobilization of the body in the Lombax orthesis, the total von Mises tensions occurring inside the body are minimal (Figure 5a). By increasing the flexion force, maximum tensions occur in the contact area between the orthesis and the body, tensions which are manifesting up to the spine (Figure 5b). In this case, high stresses are also recording in the adjacent area of the fracture, more exactly along the anterior longitudinal ligament (ALL) and in the intervertebral disc between L1 - L2 vertebrae. It can be suggested that by immobilizing the body in the Lombax orthesis, when performing a flexion movement in the sagittal plane, in the body - at the spine, occur two types of tension: tension generated by the bodice and tensions generated by the bending of the spine.

In the case of surgical stabilization of the fracture - by means of an internal spinal fixator (Figure $5 \mathrm{c}$ and $5 \mathrm{~d}$ ), the state of tension in the whole body is minimal - except for the areas adjacent to the fracture. The relative displacement of the ends of the spinal fixator is reduced, which is motive that there are appearing low intensity elastic stresses inside it - regardless of the value of the flexion or extension force. It can be noticed that at flexion forces of low magnitude occur high stresses in the adjacent area of the fracture, more precisely in the anterior ligament longitudinal (ALL) (Figure 5c). By increasing the flexion force value, the stresses in the ligament ALL disappear and occur stresses both between the L1L2 intervertebral disc and in the spinal fixator (Figure 5d).
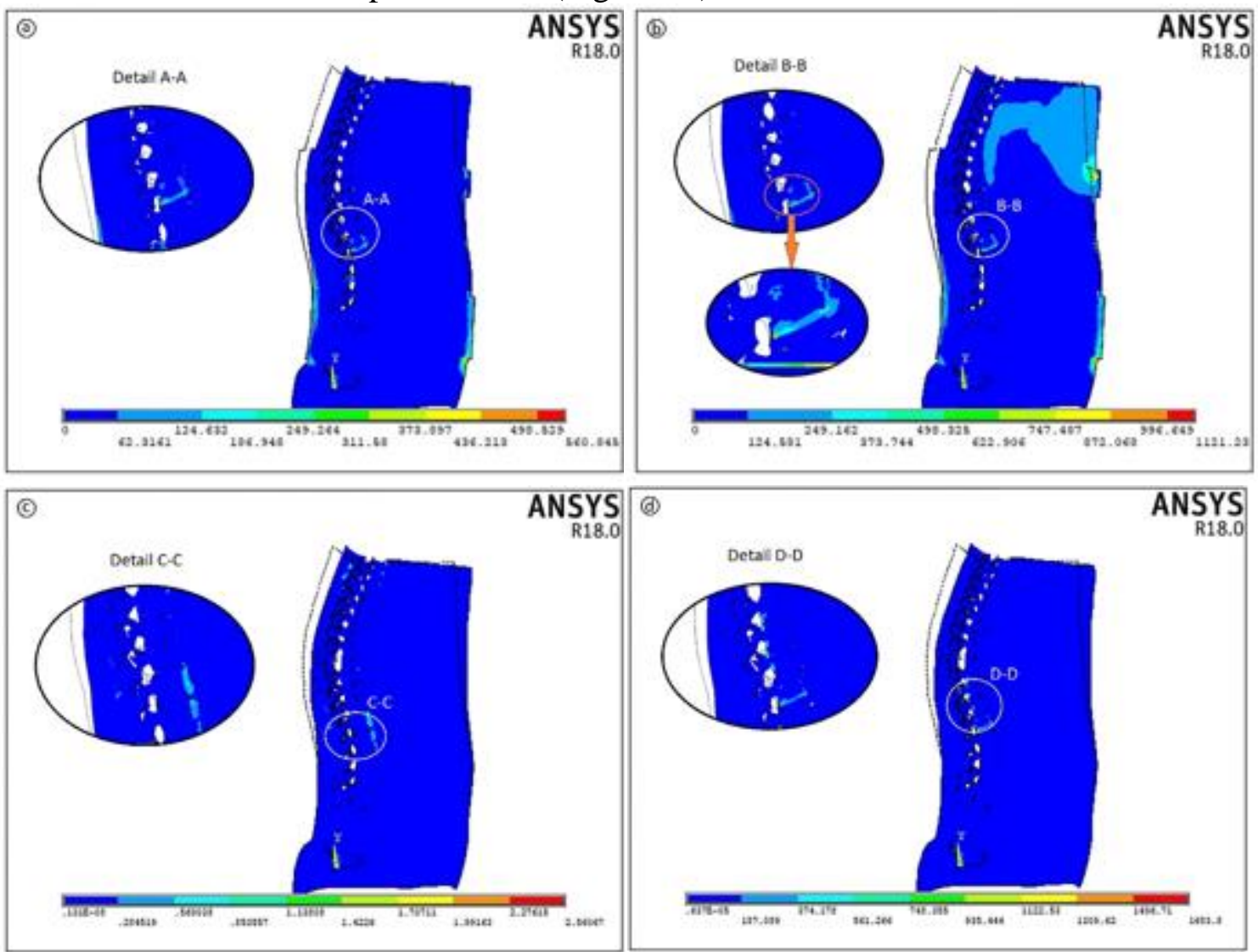

Figure 5. The variation of the total von Mises tension at the level of the body in the condition of performing a flexion motion: a) fracture stabilized with the Lombax orthesis;

b) stabilized fracture with a spinal fixator, (a, c: $F_{\text {flex }}=300 \mathrm{~N}$; b, d: $F_{\text {flex }}=700 \mathrm{~N}$ ) 
In condition of execution of the extension movement, in all cases considered, the tension inside the body is evenly distributed - with the exception of the contact area between the orthesis and the body (Figures $6 \mathrm{a}$ and $6 \mathrm{~b}$ ), or of the area adjacent to the spinal fixator (Figures. $6 \mathrm{c}$ and $6 \mathrm{~d}$ ). For the same value of the extension force, the general tensions von Mises are higher in case of immobilizing the body in the Lombax orthesis (Figure 6a and $6 \mathrm{~b}$ vs. Figures $6 \mathrm{c}$ and $6 \mathrm{~d}$ ). In condition of surgical stabilization of the fracture, high stresses appear in the L1-L2 intervertebral disc and in the spinal fixation - regardless of the value of the extension force (Figure $6 \mathrm{c}$ and Figure 6d). Knowing that the material from which the spinal fixative is made - the titanium alloy has elastic behaviour in this tension domain, it can be said that its role in the treatment of comminutive fractures is to help bring the spine back to its original position and to keep the constant distance between intervertebral discs.

From Figure 6 it can be seen that for the same type of motion (flexion or extension) the amplitude of the movements varies within very small limits. For the same value of the flexion or extension force, the amplitude of the movements performed in condition of immobilizing the body in the Lombax orthesis is lower - on average by up to $12 \%$. It can be said that body immobilization in the Lombax orthesis reduces the mobility of the body.
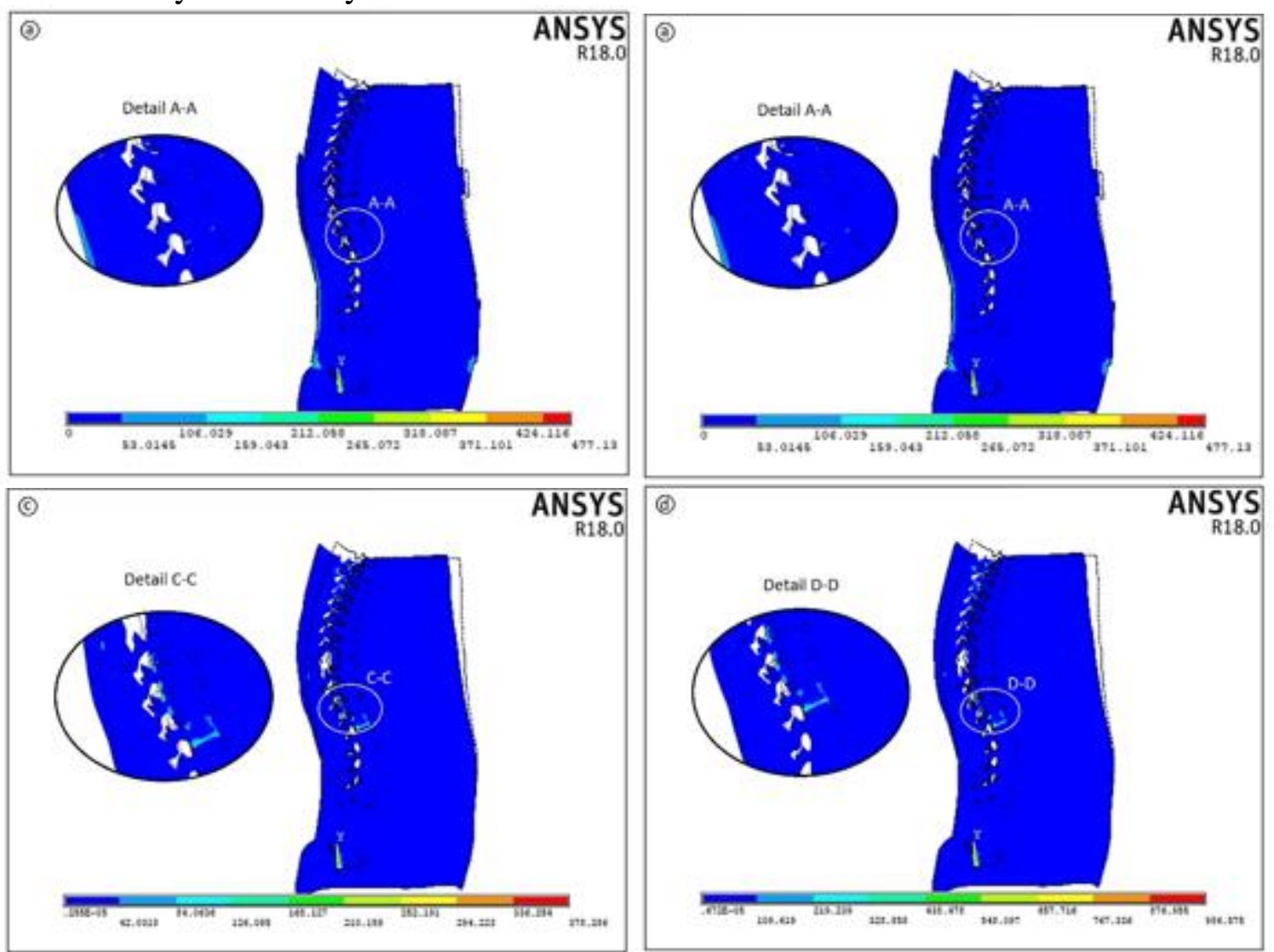

Figure 6. Variation of total von Mises tension at the body level in the condition of executing an extension movement: $\mathrm{a}, \mathrm{b}$ - the fracture stabilized with the Lombax orthesis; $\mathrm{c}, \mathrm{d}-$ the fracture stabilized by means of a spinal fixator $\left(\mathrm{a}, \mathrm{c}: \mathrm{F}_{\mathrm{ext}}=300 \mathrm{~N} ; \mathrm{b}, \mathrm{d}: \mathrm{F}_{\mathrm{ext}}=700 \mathrm{~N}\right)$

The bone fragments of the vertebra T11 (Figure 1), under the action of flexion forces or extension forces suffer displacements from equilibrium positions. In Figures 7 and 8 there are represented the displacements of the centre of gravity of each bone fragment relative to the initial position, determined along the trajectory, when the body is subjected to the flexion or extension movement. It can be seen that for the same flexion force, the displacements of the bone fragments of the traumatized vertebra T11 are higher in the condition of stabilizing the fracture by means of a spinal fixator, compared to displacements of the same bone fragments recorded under the condition of fracture immobilization in a Lombax orthesis (Figure 7a vs. Figure 7b). The size of the displacement increases with the value of the 
flexion force - regardless of the type of stabilization chosen. Under the action of the same flexion force, the fragments positioned in the anterior vertebra (fragments 1,2 and 3) record greater displacements compared to the fragments positioned in the posterior vertebra (fragments 4, 5 and 6). It may be suggested that, in the case of immobilization of the spine by means of a spinal fixator, the displacement of the bone fragments of the fractured vertebra returns to the initial position due to the elastic behaviour of the spinal fixator.
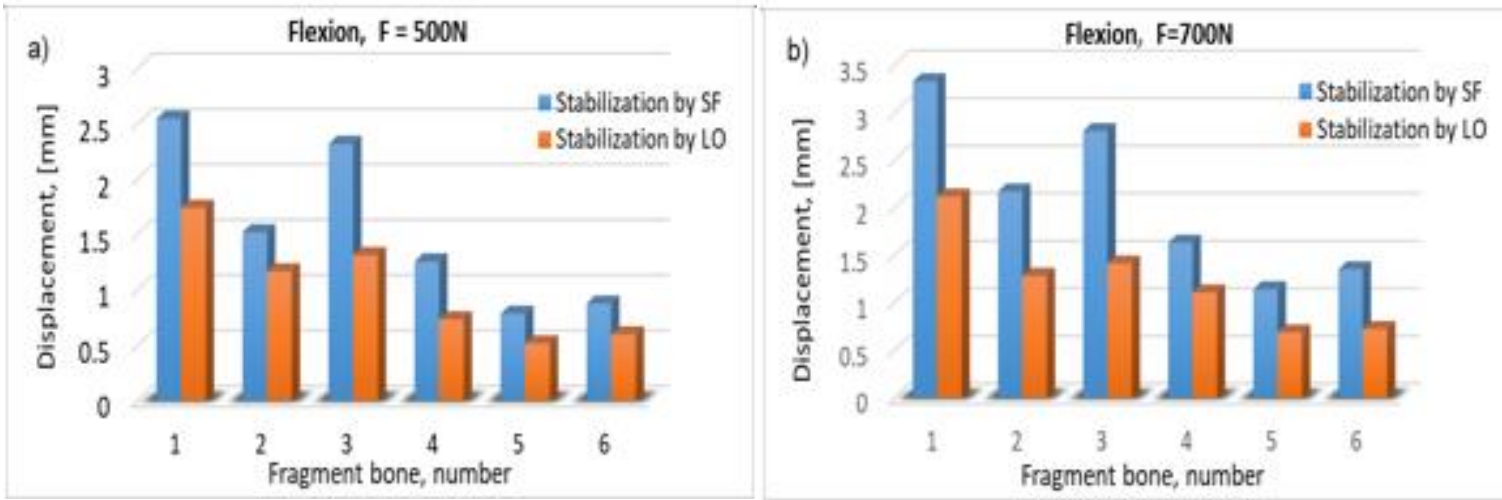

Figure 7. Average displacement of bone fragments 1- 6 of the T11 fractured vertebra during the flexion movement of the body under a force $F=500 \mathrm{~N}$ (a) or under the action of a force $\mathrm{F}=700 \mathrm{~N}$ (b)

The comparative analysis of Figure 7 and figure 8 reflects that in the case of the extension movement, the displacements of the bone fragments are much smaller - by up to $62 \%$ compared to the displacements of the same bone fragments in the flexion movement. It is noted that the displacements of the bone fragments of the traumatized vertebra T11, stabilized by immobilization in the Lombax corset, are significantly higher than the movements recorded by the same vertebral fragments - stabilized by a spinal fixator (Figure 8a and Figure 8b). The size of displacement increases with the value of extension force. However, it can be suggested that the rigid and circular stability of the Lombax corset in the thoracolumbar area doesn't allow large displacements of bone fragments in the case of flexion and extension movements.
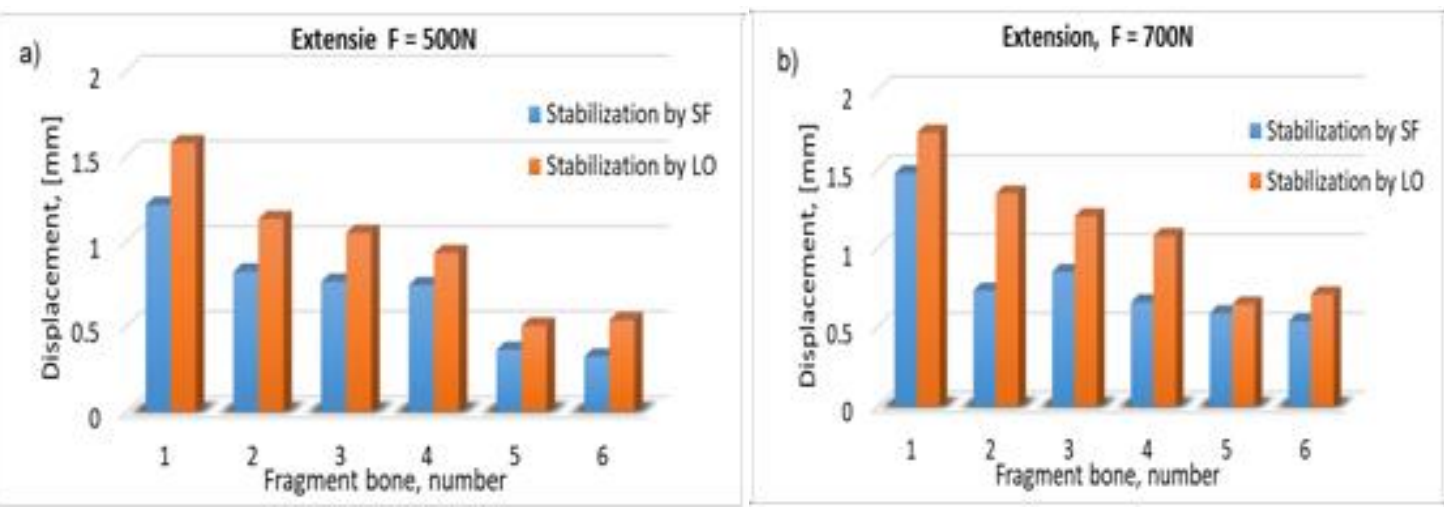

Figure 8. Average displacement of bone fragments 1-6 of the T11 fractured vertebra during the movement of the body extension under a force $F_{\text {ext }}=500 \mathrm{~N}(\mathbf{a})$ or under the action of a force $\mathrm{F}_{\mathrm{ext}}=700 \mathrm{~N}(\mathbf{b})$

\section{Results of clinical analysis}

The local kyphosis angle (LKA) and the anterior vertebral body height (AVH) of the traumatized vertebra were analysed and compared between the two groups of patients treated differently. The average results obtained after investigation on patients who have suffered trauma of burst fracture type of the T11 vertebra and which were stabilized by immobilization in Lombax orthesis - group 1 or by surgery intervention - group II are shown in Table 4. 
Table 4. Results of clinical evaluation of the patient group

\begin{tabular}{|c|c|c|c|c|c|c|c|c|c|c|}
\hline \multirow{2}{*}{ Group } & \multicolumn{3}{|c|}{$\begin{array}{c}\text { Average values of LKA after } \\
\text { (Months) }\end{array}$} & \multicolumn{3}{c|}{$\begin{array}{c}\text { Average values of AVH after } \\
\text { (Months) }\end{array}$} & \multicolumn{4}{c|}{$\begin{array}{c}\text { Average values VAS after } \\
\text { (Mouths) }\end{array}$} \\
\cline { 2 - 11 } & $\mathbf{3}$ & $\mathbf{6 s}$ & $\mathbf{1 2}$ & $\mathbf{3 s}$ & $\mathbf{6}$ & $\mathbf{1 2}$ & Initial & $\mathbf{3}$ & $\mathbf{6 s}$ & $\mathbf{1 2}$ \\
\hline $\mathrm{I}$ & $22.8^{0}$ & $27.53^{0}$ & $29.42^{0}$ & $67.4 \%$ & $62.5 \%$ & $51.2 \%$ & 9.1 & 5.3 & 4.4 & 3.4 \\
\hline II & $24.62^{0}$ & $37.43^{0}$ & $42.7^{0}$ & $73.8 \%$ & $65.4 \%$ & $63.7 \%$ & 9.2 & 4.1 & 2.9 & 2.2 \\
\hline
\end{tabular}

It can be seen that under the condition of surgical stabilization of the fracture - by inserting a spinal fixator both the height of the anterior vertebral body AVH and the local kyphosis angle (LKA) increased more pronounced. The pain indicator - VAS, has a declining trend - for both groups. It is noted that the VAS indicator value of patients from group II tends to 2 after a 12-month period - an acceptable value for patients who have suffered from spine affections. Variation in functional status of patients (ODI assessment) is presented in Figure 9.

From Figure 9 it can be seen that greater mobility of the spine presents patients undergoing surgical treatment (group II). For both groups, the functional status of the patients - carried out through the ODI assessment has a decreasing trend, stabilized under the value of 20 - considered admissible value for patients who have suffered spinal affections. It is remarked that the greater mobility of the spine is presented by patients who they were applied a surgical treatment (group II). The global vertebral mobility had greater values on the surgically treated patients.

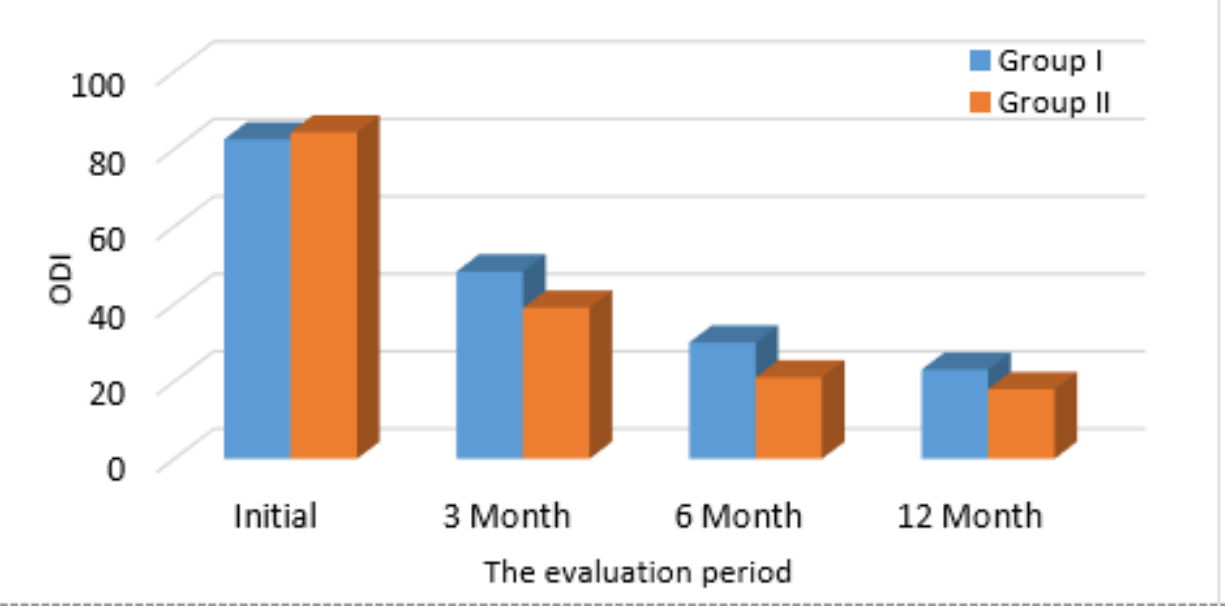

Figure 9. Functional status variation (ODI) of patients by assessment at 3,6 and 9 months

From the data presented, it is observed that there are significant differences between the condition of the patients treated by immobilisation of the body in the Lombax orthesis and that of the patients treated by surgery. Although the displacement of bone fragments is lower in patients treated by immobilization in the Lombax orthesis (Figures 7 and 8), however, the angle of local kyphosis (LKA) obtained has lower values than those determined in surgically treated patients. It can be suggested that immobilization with the Lombax orthesis limits the movement of the spine, of the bone fragments and doesn't allow their returning to the initiation position They are chaotically welding (are catching), decreasing the angle of local kyphosis (Table 4), aspect which causes the modification of geometric shape of the spine. In the case of column fractures, surgical treated, although the bone fragments are displacing over longer distances (Figure 7a and Figure 7b), they return to the initial position or in a similar position to this due to the constant maintenance of the height of the fractured vertebra and of the elasticity of the spinal fixator. This aspect allows the obtaining of a maximum kyphosis angle (Table 4). Surgical internal fixation, although more inconvenient due to the inherent complications it can generate, still allows a consolidation in the better local conditions of the vertebral body, the obtaining of lower values of the degree of pain expressed by the VAS indicator (Table 4), as well as a larger mobility of the body expressed by the ODI indicator (Figure 9). 


\section{Conclusions}

-A bidimensional model of the human body, detailed in the spine area, was conceived to study comparatively the effect created by the flexion and extension movement on a comminuted fractured vertebra and on the adjacent area - in the condition of stabilizing the fracture by immobilizing the body in the Lombax orthesis or by surgery intervention. The study was completed by observations and clinical investigations performed on groups of patients who have benefited from both treatment methods. The obtained results demonstrate the following aspects:

-by using a spinal fixator - in the case of comminutive fractures of a vertebra, respectively by applying an internal spinal fixator with pedicular screws in fractured adjacent vertebrates, it allows the maintaining of a constant height of the fractured vertebra, thus diminishing the tension state (Figures $5 \mathrm{c}$ and 5d) and remodelling to the initial position of the bone fragments of fractured vertebra. It can be suggested that although the bone fragments move from the initial positions - during the flexion or extension movement, they return to the initial position due to the elastic behaviour of the spinal fixator. These aspects allow the reconstruction of the traumatized vertebra, obtaining high values of the local kyphosis angle, of the anterior vertebral body height (AVH) (Table 4) and diminishing the state of pain;

-by using a Lombax orthesis in the treatment of comminutive fracture, burst fractures type, after a flexion movement in sagittal plane, in the body - at the spine level, occur two types of tensions: tensions created by the corset - which are manifested up to the adjacent area of the column and tensions generated by the bending of the spine (Figure 5). It can be suggested that the state of general tension contributes to maintaining pain in that area (Table 4), VAS indicator. The rigid and circular stability of the Lombax corset, in the thoraco-lumbar area doesn't allow large displacements of the bone fragments in the case of flexion and extension basic movements. However, at the end of the movement, the bone fragments don't return to a position close to the initial one, they are chaotic welded, causing vertebral deformations by decreasing the angle of local kyphosis and the anterozide height (Table 4);

-during the flexion movement respectively extension, the displacement size of the bone fragments is different depending on their position in the vertebra body, the value of the flexion force, respectively of extension, and last but not least the fracture stabilization mode (Figures 7a, 7b, 8a and Figure 8b). An increased mobility, after complete recovery, is provided by using spinal fixator (Figure 9), although this involves a longer recovery period and a high risk of specific postoperative complications.

\section{References}

1. ***http://www.wheelessonline.com/ortho/burst_frx_of_spine

2.LUND, T., NYDEGGER, T., RATHONYI, G., NOLTE, LP., SCHLENZKA, D., OXLAND, TR., Eur. Spine J., 12, Nr. 5, 2007, p.474-479.

3. OXLAND, TR., LUND, T., JOST, B., CRIPTON, P., LIPPUNER, K., JAEGER, P., NOLTE, LP., Spine, 21, Nr. 22, 1996.

4. MAGERL, F., AEBI, M., GERTZBEIN, S.D., HARMS, J., NAZARIAN, S. Eur Spine J, 3, 1994, p. 184.

5. CHOI A. P. C., ZHENG Y.P. Medical \& Biological Engineering \& Computing 2005, Vol. 43, p 259264

6. NANU, C., POEATA, I., POPESCU, C., EVA, L, TOMA, BF., TOMA, S.L., Mat Plast., 55, no. 1, 2018, p. 85.

7. WAGNAC, E, AUBIN, C.E., EL-RICH, M., Med Eng Phys., 133, no. 10, 2011, 101007.

8. IRIMICIUC, SA; AGOP, M; NICA, P; GURLUI, S; MIHAILEANU, D; TOMA S., FOCSA, C; Jpn. J. Appl. Phys., 2014, 53 (11), 173-179.

9. HIRAZI-ADL, A., AHMED, A.M., SHRIVASTAVA, S.C., Spine, 11, 1986, p. 914.

10. ROHLMANN, A., BAUER, L., ZANDER, T., BERGMANN, G., WILKE, H.J., J Biomech, 39, 2006, p. 981.

11. EL-RICH M., ARNOUX P.J., WAGNAC E., BRUNET C, AUBIN C.E., J. of Biomech., 42, 2009, p.1252-1262 
12. NAKASONE, Y., YOSHIMOTO, S., STOLARSKI, T.A., Eng. Anal. ANSYS Software. 2006, 215. 13. ROHLMANN, A., ZANDER, T., BERGMANN, G., Spine, 30, 2005, p 738.

14. QIU, T.X., TAN, K.W., LEE, V.S., TEO, E.C., Medical Engineering \&, Physics, 28, 2006, p. 656.

15. KASRA, M., SHIRAZI-ADL, A., DROUIN, G., Spine 17, 1992., 93-102.

16. OCHIA, R.S., TENCER, A.F., CHING, R.P., J. of Biomechanics, 2003, 36,1875-1881.

17. TOMA, SL, BEJINARIU, C, GHEORGHIU, DA, BACIU, C., Advanced Materials Research, 2013: 814, 173-179, DOI: 10.4028/www.scientific.net/AMR.814.173.

18. CAZAC, AM, BEJINARIU, C, BACIU, C, TOMA, SL, FLOREA, CD ; Applied Mechanics and Materials, 2014; 657:137-141 DOI: 10.4028/www.scientific.net/AMM.657.137.

19. CALIN, MA; CURTEZA, A; TOMA, SL., AGOP, M., Metalurgia International, 2013 18, p.19-22. 20. CAZAC, AM, BEJINARIU, C, IONITA, I, TOMA, SL, RODU, C., Applied Mechanics and Materials, 2014, 657: 193-197, DOI: 10.4028/www.scientific.net/AMM.657.193.

21. VIANO D.C., and LAU I.V., J. of Biomech., 1988, Vol. 21. No. 5. pp 387-399.

22. GOEL, V. K., MONROE, B. T., GILBERTSON, L. G., BRINCKMANN, P., Spine, 1995. 20(6), P.89-698

23. PANJABI MM, OXLAND T, TAKATA K, KRAG M. Spine 1993;18:1298-310.

24. HEUER, F., SCHMIDT, H., CLAES, L., WILKE, H.J., J. of Biomech. 2007, 40 (2), , p. 795-803.

25. SCHMIDT, H., KETTLER, A., HEUER, F., SIMON, U., CLAES, L., WILKE, H.-J., J. of Biomech., 2007, 32, p. 748-755

26. EE, C.K., KIM, Y.E., LEE, C.S., HONG, Y.M., JUNG, J.M., GOEL, V.K., Spine, 25, 2000, p. 2431. 27. SCHULTZ, A.B., ANDERSSON, G.B., 1981. Spine 6, 76-82.

28. BRINCKMANN, P., Spine (1986). 11 (2),149-153.

Manuscript received: 31.01 .2020 